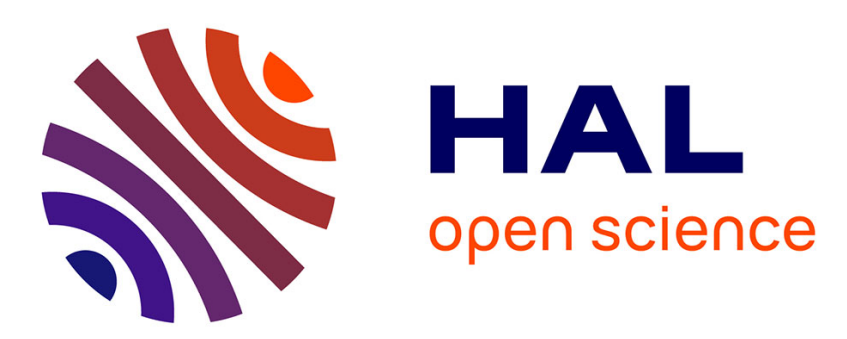

\title{
Scattering norm estimate near the threshold for energy-critical focusing semilinear wave equation
}

Thomas Duyckaerts, Frank Merle

\section{To cite this version:}

Thomas Duyckaerts, Frank Merle. Scattering norm estimate near the threshold for energy-critical focusing semilinear wave equation. 2008. hal-00300386

\section{HAL Id: hal-00300386 \\ https://hal.science/hal-00300386}

Preprint submitted on 18 Jul 2008

HAL is a multi-disciplinary open access archive for the deposit and dissemination of scientific research documents, whether they are published or not. The documents may come from teaching and research institutions in France or abroad, or from public or private research centers.
L'archive ouverte pluridisciplinaire HAL, est destinée au dépôt et à la diffusion de documents scientifiques de niveau recherche, publiés ou non, émanant des établissements d'enseignement et de recherche français ou étrangers, des laboratoires publics ou privés. 


\title{
SCATTERING NORM ESTIMATE NEAR THE THRESHOLD FOR ENERGY-CRITICAL FOCUSING SEMILINEAR WAVE EQUATION
}

\author{
THOMAS DUYCKAERTS ${ }^{1}$ AND FRANK MERLE ${ }^{2}$
}

\begin{abstract}
We consider the energy-critical semilinear focusing wave equation in dimension $N=$ $3,4,5$. An explicit solution $W$ of this equation is known. By the work of C. Kenig and F. Merle, any solution of initial condition $\left(u_{0}, u_{1}\right)$ such that $E\left(u_{0}, u_{1}\right)<E(W, 0)$ and $\left\|\nabla u_{0}\right\|_{L^{2}}<\|\nabla W\|_{L^{2}}$ is defined globally and has finite $\frac{\frac{2(N+1)}{N-2}}{L_{t, x}}$-norm, which implies that it scatters. In this note, we show that the supremum of the $L_{t, x}^{\frac{2(N+1)}{N-2}}$-norm taken on all scattering solutions at a certain level of energy below $E(W, 0)$ blows-up logarithmically as this level approaches the critical value $E(W, 0)$. We also give a similar result in the case of the radial energy-critical focusing semilinear Schrödinger equation. The proofs rely on the compactness argument of C. Kenig and F. Merle, on a classification result, due to the authors, at the energy level $E(W, 0)$, and on the analysis of the linearized equation around $W$.
\end{abstract}

\section{INTRODUCTION}

We consider the focusing energy-critical wave equation on an interval $I(0 \in I)$

$$
\left\{\begin{array}{c}
\partial_{t}^{2} u-\Delta u-|u|^{\frac{4}{N-2}} u=0, \quad(t, x) \in I \times \mathbb{R}^{N} \\
u_{\uparrow t=0}=u_{0} \in \dot{H}^{1}, \quad \partial_{t} u_{\uparrow t=0}=u_{1} \in L^{2},
\end{array}\right.
$$

where $u$ is real-valued, $N \in\{3,4,5\}, L^{2}:=L^{2}\left(\mathbb{R}^{N}\right)$ and $\dot{H}^{1}:=\dot{H}^{1}\left(\mathbb{R}^{N}\right)$. The equation (1.1) is locally well-posed in $\dot{H}^{1} \times L^{2}$ (see [Pec84], GSV92] and [SS94]): if $\left(u_{0}, u_{1}\right) \in \dot{H}^{1} \times L^{2}$, there exists an unique solution $u$, defined on a maximal time of existence $I_{\max }$ and such that for all interval $J$

$$
J \Subset I_{\max } \Longrightarrow\|u\|_{S(J)}<\infty, \text { where } S(J):=L^{\frac{2(N+1)}{N-2}}\left(J \times \mathbb{R}^{N}\right) .
$$

Furthermore, the solution $u$ of (1.1) scatters forward in time in $\dot{H}^{1} \times L^{2}$ if and only

$$
[0,+\infty) \subset I_{\max } \text { and }\|u\|_{S(0,+\infty)}<\infty .
$$

Thus the norm $S(\mathbb{R})$ measures the nonlinear effect for a given solution. The energy

$$
E\left(u(t), \partial_{t} u(t)\right)=\frac{1}{2} \int\left|\partial_{t} u(t, x)\right|^{2} d x+\frac{1}{2} \int|\nabla u(t, x)|^{2} d x-\frac{N-2}{2 N}|u(t, x)|^{\frac{2 N}{N-2}} d x
$$

is conserved for solutions of (1.1).

The defocusing case (equation (1.1) with sign + instead of - in front of the nonlinearity) has been the object of intensive studies in the last decades (see for example [SS98] and references

Date: July 18, 2008.

${ }^{1}$ Cergy-Pontoise (UMR 8088).

${ }^{2}$ Cergy-Pontoise, IHES, CNRS.

This work was partially supported by the French ANR Grant ONDNONLIN. 
therein). In this case the solutions are known to scatter, which implies, for any solution $u$, a bound of the norm $S(\mathbb{R})$ by an unspecified function of the defocusing energy

$$
E_{d}=\frac{1}{2} \int\left|\partial_{t} u\right|^{2}+\frac{1}{2} \int|\nabla u|^{2}+\frac{N-2}{2 N} \int|u|^{\frac{2 N}{N-2}} .
$$

In three spatial dimension, an explicit upper bound was proven by T. Tao [Tao06]: for any solution $u$ of the defocusing equation,

$$
\|u\|_{L_{t}^{4} L_{x}^{12}} \leq C\left(1+E_{d}\right)^{C E_{d}^{105 / 2}}
$$

which gives, by Strichartz and interpolation estimate, a similar bound for $\|u\|_{S(\mathbb{R})}$.

Going back to the focusing case, consider the explicit $\dot{H}^{1}$ stationnary solution of (1.1)

$$
W:=\frac{1}{\left(1+\frac{|x|^{2}}{N(N-2)}\right)^{\frac{N-2}{2}}} .
$$

In KM06b], C. Kenig and F. Merle have described the dynamics of (1.1) below the energy threshold $E(W, 0)$. Namely, if $E\left(u_{0}, u_{1}\right)<E(W, 0)$, then $\int\left|\nabla u_{0}\right|^{2} \neq \int|\nabla W|^{2}$ and the solution $u$ scatters (both forward and backward in time) if and only if $\int\left|\nabla u_{0}\right|^{2}<\int|\nabla W|^{2}$. This implies that for $\varepsilon>0$ the following supremum is finite:

where

$$
\mathcal{I}_{\varepsilon}=\sup _{u \in F_{\varepsilon}} \int_{\mathbb{R} \times \mathbb{R}^{N}}|u(t, x)|^{\frac{2(N+1)}{N-2}} d t d x=\sup _{u \in F_{\varepsilon}}\|u\|_{S(\mathbb{R})}^{\frac{2(N+1)}{N-2}}
$$

$$
F_{\varepsilon}:=\left\{u \text { solution of (1.1) such that } E\left(u_{0}, u_{1}\right) \leq E(W, 0)-\varepsilon^{2} \text { and } \int\left|\nabla u_{0}\right|^{2}<\int|\nabla W|^{2}\right\} \text {. }
$$

Furthermore, the existence of the non-scattering solution $W$ at the energy threshold shows that

$$
\lim _{\varepsilon \rightarrow 0^{+}} \mathcal{I}_{\varepsilon}=+\infty
$$

The purpose of this note is to give an equivalent of $\mathcal{I}_{\varepsilon}$ for small $\varepsilon$. Consider the negative eigenvalue $-\omega^{2}(\omega>0)$ of the linearized operator associated to (1.1) around $W$ :

$$
-\omega^{2}=\inf _{\substack{u \in H^{1} \\ \int u^{2}=1}} \int_{\mathbb{R}^{N}}|\nabla u|^{2}-\frac{N+2}{N-2} \int_{\mathbb{R}^{N}} W^{\frac{4}{N-2}}|u|^{2} .
$$

(See $\$ 3.1$ for details). Then

\section{Theorem 1.}

$$
\lim _{\varepsilon \rightarrow 0^{+}} \frac{\mathcal{I}_{\varepsilon}}{|\log \varepsilon|}=\frac{2}{\omega} \int_{\mathbb{R}^{N}} W^{\frac{2(N+1)}{N-2}}
$$

Remark 1.1. It would be interesting to get an explicit value of the limit $\frac{2}{\omega} \int_{\mathbb{R}^{N}} W^{\frac{2(N+1)}{N-2}}$. A straightforward computation gives:

$$
\begin{array}{ll}
\int_{\mathbb{R}^{N}} W^{\frac{2(N+1)}{N-2}}=\frac{(N(N-2))^{\frac{N}{2}}}{2^{2 N+1}} \times \frac{N !}{\left(\left(\frac{N}{2}\right) !\right)^{2}} \times \pi & \text { if } N \text { is even, } \\
\int_{\mathbb{R}^{N}} W^{\frac{2(N+1)}{N-2}}=\frac{(N(N-2))^{\frac{N}{2}}}{2} \times \frac{\left(\frac{N-1}{2}\right) !}{N !} & \text { if } N \text { is odd. }
\end{array}
$$


However we do not know any explicit expression of $\omega$.

Let us give an outline of the proof of Theorem 1. In Section 2, we show that a sequence of solutions $\left(u_{n}\right)$ such that

$$
E\left(u_{n}(0), \partial_{t} u_{n}(0)\right)<E(W, 0), \quad \int\left|\nabla u_{n}(0)\right|^{2}<\int|\nabla W|^{2} \text { and } \lim _{n \rightarrow+\infty}\left\|u_{n}\right\|_{S(\mathbb{R})}=+\infty
$$

must converge to $W$ up to modulation for a well-chosen time sequence. This relies on the compactness argument of [KM06b, Section 4], using the profile decomposition of Bahouri-Gérard BG99], and on the classification of the solutions of (1.1) at the threshold of energy in our previous work DM07b]. The second step of the proof is an analysis of the behaviour of solutions whose initial conditions are close to $(W, 0)$, which is carried out in Section 3. We show, as a consequence of the existence of the negative eigenvalue $-\omega^{2}$, that such solutions go away from the solution $W$ in a time which is of logarithmic order with respect to the distance of the initial condition to $(W, 0)$. In Section 1 we put together the preceding arguments to prove Theorem 1 .

Our arguments do not depend strongly on the nature of equation (1.1), and we except that a logarithmic estimate of the scattering norm $S(\mathbb{R})$ near the threshold holds in similar situations, as long as the linearized operator around the ground state admits real nonzero eigenvalues. In Section 5 we give a result and a sketch of proof in the case of the radial, energy-critical focusing nonlinear Schrödinger equation.

\section{Convergence to $W$ And $W^{-}$NeAr the threshold}

In all the article, we will denote by $\|\cdot\|_{p}$ the $L^{p}$ norm on $\mathbb{R}^{N}$.

Equation (1.1) enjoys the following invariances: if $u$ is a solution and $t_{0} \in \mathbb{R}, x_{0} \in \mathbb{R}^{N}, \lambda_{0}>0$, $\delta_{0}, \delta_{1} \in\{-1,+1\}$, then

$$
v(t, x)=\frac{\delta_{0}}{\lambda_{0}^{(N-2) / 2}} u\left(\frac{t_{0}+\delta_{1} t}{\lambda_{0}}, \frac{x+x_{0}}{\lambda_{0}}\right)
$$

is also a solution. Note that the energy of $u$ and, if $u$ is globally defined, the norm $\|u\|_{S(\mathbb{R})}$ are not changed by these transformations.

We recall the following classification Theorem, proven in KM06b, for the case $E\left(u_{0}, u_{1}\right)<$ $E(W, 0)$, and in [DM07b] for the existence of $W^{-}$and the case $E\left(u_{0}, u_{1}\right)=E(W, 0)$ :

Theorem A (Kenig-Merle,Duyckaerts-Merle). There exists a global solution $W^{-}$of (1.1) such that

$$
\begin{gathered}
E\left(W^{-}(0), \partial_{t} W^{-}(0)\right)=E(W, 0), \quad\left\|\nabla W_{-}(0)\right\|_{2}<\|\nabla W\|_{2} \\
\left\|W^{-}\right\|_{S(-\infty, 0)}<\infty, \quad \lim _{t \rightarrow+\infty}\left\|\nabla\left(W^{-}(t)-W\right)\right\|_{2}+\left\|\partial_{t}\left(W^{-}(t)-W\right)\right\|_{2}=0 .
\end{gathered}
$$

Moreover, if $u$ is a solution of (1.1) such that $E\left(u_{0}, u_{1}\right) \leq E(W, 0)$ and $\left\|\nabla u_{0}\right\|_{2} \leq\|\nabla W\|_{2}$, then $u$ is globally defined. If furthermore $\|u\|_{S(\mathbb{R})}=\infty$, then $u=W^{-}$or $u=W$ up to the invariances of the equation.

We will also need the following simple version of long-time perturbation theory results (see e.g. KM06b, Theorem 2.20]).

Lemma 2.1. Let $M>0$. Then there exist positive constants $\varepsilon(M)$ and $C(M)$ such that for all solutions $v$ and $u$ of (1.1), with initial conditions $\left(v_{0}, v_{1}\right)$ and $\left(u_{0}, u_{1}\right)$, if the forward time of 
existence of $v$ is infinite and

$$
\|v\|_{S(0,+\infty)} \leq M \text { and }\left\|\nabla\left(u_{0}-v_{0}\right)\right\|_{2}+\left\|u_{1}-v_{1}\right\|_{2} \leq \varepsilon(M),
$$

then $u$ is globally defined for positive times and $\|u\|_{S(0,+\infty)} \leq C(M)$. A similar statement holds for negative times.

In this section we show the following:

Proposition 2.2. Let $u_{n}$ be a family of solutions of (1.1), such that

$$
E\left(u_{n}(0), \partial_{t} u_{n}(0)\right)<E(W, 0), \quad\left\|\nabla u_{n}(0)\right\|_{2}<\|\nabla W\|_{2} .
$$

and $\lim _{n \rightarrow+\infty}\left\|u_{n}\right\|_{S(\mathbb{R})}=+\infty$. Let $\left(t_{n}\right)_{n}$ be a time sequence.

(a) Assume

$$
\lim _{n \rightarrow+\infty}\left\|u_{n}\right\|_{S\left(-\infty, t_{n}\right)}=\lim _{n \rightarrow+\infty}\left\|u_{n}\right\|_{S\left(t_{n},+\infty\right)}=+\infty .
$$

Then, up to the extraction of a subsequence there exist $\delta_{0} \in\{-1,+1\}$ and sequences of parameters $x_{n} \in \mathbb{R}^{n}, \lambda_{n}>0$ such that

$$
\lim _{n \rightarrow+\infty}\left\|\frac{\delta_{0}}{\lambda_{n}^{N / 2}} \nabla u_{n}\left(t_{n}, \frac{\cdot-x_{n}}{\lambda_{n}}\right)-\nabla W\right\|_{2}+\left\|\frac{\partial u_{n}}{\partial t}\left(t_{n}\right)\right\|_{2}=0 .
$$

(b) Assume that there exists $C_{0} \in(0,+\infty)$ such that

$$
\lim _{n \rightarrow+\infty}\left\|u_{n}\right\|_{S\left(-\infty, t_{n}\right)}=+\infty \text { and } \lim _{n \rightarrow+\infty}\left\|u_{n}\right\|_{S\left(t_{n},+\infty\right)}=C_{0} .
$$

Then, up to the extraction of a subsequence there exist $t_{0} \in \mathbb{R}, \delta_{0}, \delta_{1} \in\{-1,+1\}$, and sequences of parameters $x_{n} \in \mathbb{R}^{n}, \lambda_{n}>0$ such that

$$
\begin{aligned}
\lim _{n \rightarrow+\infty}\left\|\frac{\delta_{0}}{\lambda_{n}^{N / 2}} \nabla u_{n}\left(t_{n}, \frac{\cdot-x_{n}}{\lambda_{n}}\right)-\nabla W^{-}\left(t_{0}\right)\right\|_{2} \\
+\left\|\frac{\delta_{0}}{\lambda_{n}^{N / 2}} \frac{\partial u_{n}}{\partial t}\left(t_{n}, \frac{\cdot-x_{n}}{\lambda_{n}}\right)-\frac{\partial W^{-}}{\partial t}\left(t_{0}\right)\right\|_{2}=0 .
\end{aligned}
$$

Remark 2.3. Case (B) will not be used in the proof of Theorem 1, and is stated only for its own interest.

Sketch of Proof. We will sketch the proof (a), the proof of (b) is similar and left to the reader. Translating in time all the $u_{n}$, we may assume that $t_{n}=0$ for all $n$, and thus

$$
\lim _{n \rightarrow+\infty}\left\|u_{n}\right\|_{S(-\infty, 0)}=\lim _{n \rightarrow+\infty}\left\|u_{n}\right\|_{S(0,+\infty)}=+\infty .
$$

In view of (2.1) and (2.2), one can show, using the profile decomposition of [BG99] as in KM06b, Proposition 4.2], that there exist (up to the extraction of a subsequence) parameters $\lambda_{n}>0, x_{n} \in \mathbb{R}^{N}$, and functions $\left(v_{0}, v_{1}\right) \in \dot{H}^{1} \times L^{2}$ such that

$$
\lim _{n \rightarrow+\infty}\left\|\frac{1}{\lambda_{n}^{N / 2}} \nabla u_{n}\left(0, \frac{\cdot-x_{n}}{\lambda_{n}}\right)-\nabla v_{0}\right\|_{2}+\left\|\frac{1}{\lambda_{n}^{N / 2}} \frac{\partial u_{n}}{\partial t}\left(0, \frac{\cdot-x_{n}}{\lambda_{n}}\right)-v_{1}\right\|_{2}=0 .
$$

We refer to [KM06a, Section 4] and also [DM07a, Lemma 2.5] for proofs in the case of nonlinear Schrödinger equations that readily apply to our case. Note that

$$
\left\|\nabla v_{0}\right\|_{2} \leq\|\nabla W\|_{2}, \quad E\left(v_{0}, v_{1}\right) \leq E(W, 0) .
$$


Let $v$ be the solution of (1.1) with initial conditions $\left(v_{0}, v_{1}\right)$. Theorem $\mathrm{A}$ and (2.3) imply that $v$ is globally defined. By Lemma 2.1 and by (2.2),

$$
\|v\|_{S(-\infty, 0)}=+\infty, \quad\|v\|_{S(0,+\infty)}=+\infty \text {. }
$$

This shows, again by Theorem A, that $v=W$, up to the invariances of equation (1.1) concluding the proof.

\section{Estimates NEAR THE THRESHOLD}

3.1. Preliminaries on the linearized equation. In this subsection, we recall results on the linearized equation near $W$. We refer to [DM07b] for the details. Let $u$ be a solution of (1.1) which is close to $W$. Write $u=W+h$. Then $h$ is solution to the equation

$$
\begin{gathered}
\left(\partial_{t}^{2}+L\right) h=R(h) . \\
L:=-\Delta-\frac{N+2}{N-2} W^{\frac{4}{N-2}}, \quad R(h):=|W+h|^{\frac{4}{N-2}}(W+h)-W^{\frac{N+2}{N-2}}-\frac{N+2}{N-2} W^{\frac{4}{N-2}} h .
\end{gathered}
$$

Let

$$
W_{0}=a\left(\frac{N-2}{2} W+x \cdot \nabla W\right), \quad W_{j}=b \partial_{x_{j}} W, j=1 \ldots N .
$$

where the constants $a$ and $b$ are chosen so that $\left\|\nabla W_{j}\right\|_{2}=1$ for $j=0,1, \ldots, N$. By the invariances of equation (1.1), $L\left(W_{j}\right)=0$ for $j=0, \ldots, N$. As a consequence the functions $W_{j}$, $j=0, \ldots, N$ are in the kernel of the quadratic form

$$
Q(h)=\frac{1}{2} \int L h h=\frac{1}{2} \int|\nabla h|^{2}-\frac{N+2}{2(N-2)} \int W^{\frac{4}{N-2}} h^{2} .
$$

Observe that if $\left(h, \partial_{t} h\right)$ is small in $\dot{H}^{1} \times L^{2}$,

$$
E\left(W+h, \partial_{t} h\right)=E(W, 0)+Q(h)+\frac{1}{2} \int\left|\partial_{t} h\right|^{2}+O\left(\|h\|_{\frac{2 N}{N-2}}^{3}\right) .
$$

Furthermore, one can check that the infimum of $Q(h)$ for $h \in H^{1},\|h\|_{L^{2}}=1$, is negative, and thus that $L$ admits a positive, radial eigenfunction $\mathcal{Y}$ with eigenvalue $-\omega^{2}<0$. We normalize $\mathcal{Y}$ such that $\int \mathcal{Y}^{2}=1$. The self-adjointness of $L$ implies

$$
\int \mathcal{Y} W_{j}=0, \quad j=0 \ldots N
$$

Consider

$$
G_{\perp}:=\left\{f \in \dot{H}^{1}, \int \mathcal{Y} f=\int \nabla W_{0} \cdot \nabla f=\ldots=\int \nabla W_{N} \cdot \nabla f=0\right\} .
$$

The following result (see Proposition 5.5 of DM07b) shows in particular that $-\omega^{2}$ is the only negative eigenvalue of $L$ :

Claim 3.1. There exists a constant $c_{Q}>0$ such that

$$
\forall h \in G_{\perp}, \quad Q(h) \geq c_{Q}\|\nabla h\|_{L^{2}}^{2} .
$$

As a consequence of the Strichartz estimates for the linear wave equation (see [GV95] and [LS95]), we easily get the following Strichartz-type estimate for equation (3.1). 
Claim 3.2. There exist constants $\tilde{c}, C>0$ such that if $h$ is a solution of (3.1) on an interval $\left[t_{0}, t_{1}\right]$ such that

$$
\|\nabla h\|_{2}+\left\|\partial_{t} h\right\|_{2}+\left|t_{0}-t_{1}\right| \leq \tilde{c}
$$

Then

$$
\|h\|_{S\left(t_{0}, t_{1}\right)} \leq C\left(\left\|\nabla h\left(t_{0}\right)\right\|_{2}+\left\|\partial_{t} h\left(t_{0}\right)\right\|_{2}\right) .
$$

3.2. Estimate on the exit time. In this subsection, we consider a sequence $u_{n}$ of solutions of (1.1) such that

$$
\begin{gathered}
\lim _{n \rightarrow+\infty}\left\|\nabla\left(u_{n}(0)-W\right)\right\|_{2}+\left\|\partial_{t} u_{n}(0)\right\|_{2}=0 \\
E(W, 0)-E\left(u_{n}, \partial_{t} u_{n}\right)=\varepsilon_{n}^{2} \underset{n \rightarrow+\infty}{\longrightarrow} 0 \text { and } \forall n>0,\left\|\nabla u_{n}(0)\right\|_{2}<\|\nabla W\|_{2} .
\end{gathered}
$$

Let $h_{n}=u_{n}-W$ and decompose $h_{n}$ as

$$
h_{n}(t)=\beta_{n}(t) \mathcal{Y}+\sum_{j=0}^{N} \gamma_{j, n}(t) W_{j}+g_{n}(t), \quad g_{n}(t) \in G_{\perp} .
$$

For this, observe that the condition $g_{n}(t) \in G_{\perp}$ is equivalent to

$$
\begin{gathered}
\beta_{n}(t)=\int h_{n}(t) \mathcal{Y}, \\
\gamma_{0, n}(t)=\int \nabla\left(h_{n}(t)-\beta_{n}(t) \mathcal{Y}\right) \cdot \nabla W_{0}, \quad \gamma_{j, n}(t)=\int \nabla h_{n}(t) \cdot \nabla W_{j}, j=1, \ldots, N .
\end{gathered}
$$

(we used that $\mathcal{Y}$ and $W$ being radial, $\int \nabla \mathcal{Y} \cdot \nabla W_{j}=b \int \nabla \mathcal{Y} \cdot \partial_{x_{j}} \nabla W=0$ if $j \in\{1, \ldots, N\}$ and by a similar argument, $\int \nabla W_{j} \cdot \nabla W_{k}=0$ if $j \neq k$ ). We have:

Claim 3.3. Assume (3.5). Then there exists sequences $\lambda_{n} \in(0,+\infty), x_{n} \in \mathbb{R}^{N}$ such that

$$
\lim _{n \rightarrow+\infty} \lambda_{n}=1, \quad \lim _{n \rightarrow+\infty} x_{n}=0,
$$

and for all $n$, noting $c_{0}=\int \nabla \mathcal{Y} \cdot \nabla W_{0}$,

$$
\begin{gathered}
\frac{1}{\lambda_{n}^{\frac{N}{2}}} \int \nabla u_{n}\left(\frac{x-x_{n}}{\lambda_{n}}\right) \cdot \nabla W_{0}(x) d x-\frac{c_{0}}{\lambda_{n}^{\frac{N-2}{2}}} \int\left(u_{n}\left(\frac{x-x_{n}}{\lambda_{n}}\right)-W(x)\right) \mathcal{Y}(x) d x=0 \\
\forall j \in\{1, \ldots, N\}, \quad \frac{1}{\lambda_{n}^{\frac{N}{2}}} \int \nabla u_{n}\left(\frac{x-x_{n}}{\lambda_{n}}\right) \cdot \nabla W_{j}(x)=0 .
\end{gathered}
$$

Sketch of proof. Consider the mapping $J:(\lambda, X, u) \mapsto\left(J_{0}, J_{1}, \ldots, J_{N}\right)$ where

$$
\begin{aligned}
& J_{0}=\frac{1}{\lambda^{\frac{N}{2}}} \int \nabla u\left(\frac{x-X}{\lambda}\right) \cdot \nabla W_{0}(x) d x-\frac{c_{0}}{\lambda^{\frac{N-2}{2}}} \int\left(u\left(\frac{x-X}{\lambda}\right)-W(x)\right) \mathcal{Y}(x) d x \\
& J_{k}=\frac{1}{\lambda^{\frac{N}{2}}} \int \nabla u\left(\frac{x-X}{\lambda}\right) \cdot \nabla W_{k}(x), \quad k \in\{1, \ldots, N\} .
\end{aligned}
$$

A straightforward computation shows that $J=0$ and $\left(\frac{\partial J}{\partial \lambda}, \frac{\partial J}{\partial X_{1}}, \ldots, \frac{\partial J}{\partial X_{N}}\right)$ is diagonal and invertible at the point $(1,0, \ldots, 0, W)$. The Claim then follows from (3.5) and the implicit function theorem. 
Observe that the conditions (3.10) and (3.11) are equivalent, by (3.8) and (3.9), to the condition that the parameters $\gamma_{j n}$ corresponding to the modulated solution $\frac{1}{\lambda_{n}^{\frac{N-2}{2}}} u_{n}\left(\frac{t}{\lambda_{n}}, \frac{x}{\lambda_{n}}\right)$ vanish at $t=0$. By Claim 3.3 we can assume, up to translation and scaling, that

$$
\forall n, \forall j \in\{0,1, \ldots, N\}, \quad \gamma_{j, n}(0)=0 .
$$

The main result of this subsection is the following:

Proposition 3.4. There exist a constant $\eta_{0}$, such that for all $\eta \in\left(0, \eta_{0}\right)$, for all sequence $\left(u_{n}\right)$ satisfying (3.5), (3.6), (3.12) and such that $\beta_{n}(0) \beta_{n}^{\prime}(0) \geq 0$, if

$$
T_{n}(\eta)=\inf \left\{t \geq 0:\left|\beta_{n}(t)\right| \geq \eta\right\},
$$

then for large $n, \beta_{n}(0) \neq 0, T_{n}(\eta) \in(0,+\infty)$ and

$$
\lim _{n \rightarrow+\infty} \frac{T_{n}(\eta)}{|\log | \beta_{n}(0)||}=\frac{1}{\omega} .
$$

Furthermore,

$$
\liminf _{n \rightarrow+\infty}\left|\beta_{n}^{\prime}\left(T_{n}(\eta)\right)\right| \geq \omega \eta
$$

Remark 3.5. If $\beta_{n}(0) \beta_{n}^{\prime}(0)<0$, we may achieve the condition $\beta_{n}(0) \beta_{n}^{\prime}(0) \geq 0$ by considering the solution $u_{n}(-t, x)$ instead of $u_{n}(t, x)$.

The remainder of this subsection is devoted to the proof of Proposition 3.4 . We first give, as a consequence of the orthogonality conditions (3.12), a purely variational lower bound on $\left|\beta_{n}(0)\right|$ (Claim 3.6). We then give (Lemma 3.7) precise estimates on $\beta_{n}(t)$ and $\left\|\partial_{t} h_{n}(t)\right\|_{2}+\left\|\nabla h_{n}(t)\right\|_{2}$, on an interval $\left(0, t_{n}\right)$ where a priori bounds are assumed. These estimates will give the desired bounds on the exit time $T_{n}(\eta)$. We will write:

$$
\left\|\partial_{t, x} h_{n}(t)\right\|_{2}=\left\|\nabla h_{n}(t)\right\|_{2}+\left\|\partial_{t} h_{n}(t)\right\|_{2} .
$$

Claim 3.6. There exists $M_{0}>0$ such that for all sequence $\left(u_{n}\right)$ of solutions of (1.1) satisfying (3.5), (3.6) and (3.12) we have

$$
\beta_{n}(0) \neq 0 \text { and } \limsup _{n \rightarrow+\infty} \frac{\left\|\partial_{t, x} h_{n}(0)\right\|_{2}+\varepsilon_{n}}{\left|\beta_{n}(0)\right|} \leq M_{0} .
$$

Proof. Developping the energy as in (3.3), we get

$$
E(W, 0)-\varepsilon_{n}^{2}=E\left(W+h_{n}, \partial_{t} h_{n}\right)=E(W, 0)+Q\left(h_{n}\right)+\frac{1}{2}\left\|\partial_{t} h_{n}\right\|_{2}^{2}+O\left(\left\|\nabla h_{n}\right\|_{2}^{3}\right) .
$$

The expression (3.7) of $h$ at $t=0$ yields, in view of (3.12)

$$
\left\|\nabla h_{n}(0)\right\|_{2} \leq C\left(\left|\beta_{n}(0)\right|+\left\|\nabla g_{n}(0)\right\|_{2}\right) .
$$

Furthermore, taking into account that $Q(\mathcal{Y})<0$ and that the functions $W_{j}$ are in the kernel of $Q$ for $j=0 \ldots N$, we get

$$
Q\left(h_{n}(0)\right)=-\beta_{n}^{2}(0)|Q(\mathcal{Y})|+Q\left(g_{n}(0)\right) .
$$

Combining the preceding estimates, we obtain

$$
\beta_{n}^{2}(0)|Q(\mathcal{Y})|=\varepsilon_{n}^{2}+Q\left(g_{n}(0)\right)+\frac{1}{2}\left\|\partial_{t} h_{n}(0)\right\|_{2}^{2}+O\left(\beta_{n}^{3}(0)+\left\|\nabla g_{n}(0)\right\|_{2}^{3}\right) .
$$


By Claim 3.1, $Q\left(g_{n}(0)\right) \geq c_{Q}\left\|\nabla g_{n}(0)\right\|_{2}^{2}$. This yields for large $n$,

$$
2 \beta_{n}^{2}(0)|Q(\mathcal{Y})| \geq \varepsilon_{n}^{2}+c_{Q}\left\|\nabla g_{n}(0)\right\|_{2}^{2}+\frac{1}{2}\left\|\partial_{t} h_{n}(0)\right\|_{2}^{2} \geq \varepsilon_{n}^{2}+c\left\|\nabla h_{n}(0)\right\|_{2}^{2}+\frac{1}{2}\left\|\partial_{t} h_{n}(0)\right\|_{2}^{2},
$$

which concludes the proof of the claim.

Our next result is the following Lemma:

Lemma 3.7 (Growth on $\left[0, T_{n}\right]$ ). Let us fix $\omega^{+}$and $\omega^{-}$, close to $\omega$, such that $\omega^{-}<\omega<\omega^{+}$. There exist positive constants $\tau_{0}, K_{0}$ (depending only on the choice of $\omega_{ \pm}$) with the following property. Let $\left(u_{n}\right)_{n}$ be a sequence of solutions of (1.1) satisfying (3.5), (3.6) and such that $\beta_{n}(0) \beta_{n}{ }^{\prime}(0) \geq 0$. Let $M>M_{0}$ (where $M_{0}$ is given by Claim 3.6). Let $\eta$ such that

$$
0<\eta<\frac{1}{K_{0} M^{3}} .
$$

Define

$$
t_{n}=t_{n}(M, \eta)=\inf \left\{t \geq 0:\left\|\partial_{t, x} h_{n}(t)\right\|_{2} \geq M\left|\beta_{n}(t)\right| \text { or }\left|\beta_{n}(t)\right| \geq \eta\right\} .
$$

Then there exists $\tilde{n}>0$ such that for $n \geq \tilde{n}$,

$$
\begin{aligned}
& \forall t \in\left[\tau_{0}, t_{n}\right), \quad \omega^{-}\left|\beta_{n}(t)\right| \leq\left|\beta_{n}^{\prime}(t)\right| \leq \omega^{+}\left|\beta_{n}(t)\right| \\
& \forall t \in\left[\tau_{0}, t_{n}\right), \quad \frac{1}{K_{0}}\left|\beta_{n}(0)\right| e^{\omega^{-} t} \leq\left|\beta_{n}(t)\right| \leq K_{0}\left|\beta_{n}(0)\right| e^{\omega^{+} t} \\
& \forall t \in\left[0, t_{n}\right), \quad\left\|\partial_{t, x} h_{n}(t)\right\|_{2} \leq K_{0}\left|\beta_{n}(t)\right| .
\end{aligned}
$$

Before proving the lemma, we will show that it implies Proposition 3.4. For this we take $M=1+\max \left\{M_{0}, K_{0}\right\}$ and apply Lemma 3.7. Then by (3.19), $\left\|\partial_{t, x} h_{n}(t)\right\|_{2}<M\left|\beta_{n}(t)\right|$ on $\left[0, t_{n}\right]$ and thus

$$
t_{n}(\eta, M)=\inf \left\{t \geq 0:\left|\beta_{n}(t)\right| \geq \eta\right\}=T_{n}(\eta) .
$$

This shows by (3.18) that $T_{n}(\eta) \in(0,+\infty)$ for large $n$, and by continuity of $\beta_{n}$, that $\beta_{n}\left(T_{n}(\eta)\right)=$ $\eta$. In particular, $T_{n}(\eta)$ must tend to infinity; otherwise, as $\beta_{n}(0)$ tends to 0 , the continuity of the flow would imply that $u_{n}\left(T_{n}(\eta)\right)$ tends to $W$ and $\beta_{n}\left(T_{n}(\eta)\right)$ to 0 , a contradiction. By (3.18), we get for large $n$,

$$
\frac{1}{K_{0}}\left|\beta_{n}(0)\right| e^{\omega^{-} T_{n}(\eta)} \leq \eta \leq K_{0}\left|\beta_{n}(0)\right| e^{\omega^{+} T_{n}(\eta)} .
$$

By the upper bound inequality we get (noticing that $\log \left|\beta_{n}(0)\right|$ is negative for large time)

$$
|\log | \beta_{n}(0)||+\log \eta \leq \log \left(K_{0}\right)+\omega^{+} T_{n}(\eta) .
$$

Hence, using that $\beta_{n}(0)$ tends to 0 , as $n$ goes to infinity,

Letting $\omega_{+}$tends to $\omega$ we get

$$
\frac{1}{\omega^{+}} \leq \liminf _{n \rightarrow+\infty} \frac{T_{n}(\eta)}{|\log | \beta_{n}(0) \mid}
$$

$$
\frac{1}{\omega} \leq \liminf _{n \rightarrow+\infty} \frac{T_{n}(\eta)}{|\log | \beta_{n}(0)||}
$$

By the same argument, we get

$$
\limsup _{n \rightarrow+\infty} \frac{T_{n}(\eta)}{|\log | \beta_{n}(0) \mid} \leq \frac{1}{\omega}
$$


which concludes the proof of (3.13).

To conclude the proof of Proposition 3.4 observe that (3.17) implies, for large $n$,

$$
\omega^{-} \eta=\omega^{-}\left|\beta_{n}\left(T_{n}(\eta)\right)\right| \leq\left|\beta_{n}^{\prime}\left(T_{n}(\eta)\right)\right|,
$$

which yields (3.14).

In the remainder of this subsection we prove Lemma 3.7.

Proof of Lemma 3.7. In view of Claim 3.6, the fact that $\beta_{n}(0)$ tends to 0 and the continuity of $\beta_{n}$ and $\left\|\partial_{t, x} h_{n}\right\|_{2}$, the time $t_{n}$ is strictly positive. Furthermore,

$$
\begin{aligned}
& \forall n, \forall t \in\left(0, t_{n}\right), \quad\left|\beta_{n}(t)\right| \leq \eta \\
& \forall n, \forall t \in\left(0, t_{n}\right), \quad\left\|\partial_{t, x} h_{n}(t)\right\|_{2} \leq M\left|\beta_{n}(t)\right| .
\end{aligned}
$$

Proof of (3.17).

Let

$$
m=\frac{1}{2} \min \left\{\omega^{2}-\left(\omega^{-}\right)^{2},\left(\omega^{+}\right)^{2}-\omega^{2}\right\} .
$$

We first show that if $\eta$ satisfies (3.15), then

$$
\left|\beta_{n}^{\prime \prime}-\omega^{2} \beta_{n}\right| \leq m\left|\beta_{n}(t)\right| \text {. }
$$

Differentiating twice the equality $\beta_{n}=\int h_{n} \mathcal{Y}$, we get, by equation (3.1),

$$
\beta_{n}{ }^{\prime \prime}-\omega^{2} \beta_{n}=\int \partial_{t}^{2} h_{n} \mathcal{Y}-\omega^{2} \int h_{n} \mathcal{Y}=\int R\left(h_{n}\right) \mathcal{Y}-\int\left(L h_{n}+\omega^{2} h_{n}\right) \mathcal{Y}=\int R\left(h_{n}\right) \mathcal{Y}
$$

Thus there exists a constant $C_{1}$, independent of all parameters, such that

$$
\left|\beta_{n}^{\prime \prime}-\omega^{2} \beta_{n}\right| \leq C_{1}\left\|\partial_{t, x} h_{n}\right\|_{2}^{2}
$$

By $(3.20)$ and 3.21

$$
\left|\beta_{n}^{\prime \prime}-\omega^{2} \beta_{n}\right| \leq C_{1} M^{2} \beta_{n}^{2} \leq C_{1} M^{2} \eta\left|\beta_{n}\right|
$$

which yields, if $C_{1} M^{2} \eta \leq m$ (which follows from (3.15) if $K_{0}$ is large enough), the desired estimate (3.22).

In what follows, we will assume that $\beta_{n}(0) \geq 0$ and $\beta_{n}{ }^{\prime}(0) \geq 0$ (otherwise, replace $\beta_{n}$ by $-\beta_{n}$ in the forthcoming argument). We next show that for $t \in\left(0, t_{n}\right)$,

$$
\beta_{n}^{\prime \prime}(t)>0, \quad \beta_{n}{ }^{\prime}(t)>0, \quad \beta_{n}(t)>0 .
$$

Indeed by (3.22),

$$
\left(\omega^{2}-m\right) \beta_{n}(t) \leq \beta_{n}{ }^{\prime \prime}(t) \leq\left(\omega^{2}+m\right) \beta_{n}(t) .
$$

As $\beta_{n}(0)>0$ by Claim 3.6, we get that $\beta, \beta^{\prime}$ and $\beta^{\prime \prime}$ are (strictly) positive for small positive $t$. This shows that 3.24 holds near 0 , and by an elementary monotonicity argument, that it holds for all $t \in\left(0, t_{n}\right]$.

We are now ready to show (3.17). For this we write, as a consequence of (3.25)

$$
\left(\beta_{n}^{\prime}-\omega^{-} \beta_{n}\right)^{\prime}=\beta_{n}^{\prime \prime}-\omega^{-} \beta_{n}^{\prime} \geq-\omega^{-}\left(\beta_{n}^{\prime}-\omega^{-} \beta_{n}\right)+\left(\omega^{2}-\left(\omega^{-}\right)^{2}-m\right) \beta_{n} \geq-\omega^{-}\left(\beta_{n}^{\prime}-\omega^{-} \beta_{n}\right)+m \beta_{n} .
$$

Hence (using that $\beta_{n}$ increases with time)

$$
\frac{d}{d t}\left[e^{\omega^{-} t}\left(\beta_{n}^{\prime}-\omega^{-} \beta_{n}\right)\right] \geq m e^{\omega^{-} t} \beta_{n}(0) .
$$


Integrating between 0 and $t$ we get

$$
e^{\omega^{-} t}\left(\beta_{n}^{\prime}-\omega^{-} \beta_{n}\right) \geq m \beta_{n}(0) \int_{0}^{t} e^{\omega^{-} s} d s+\beta_{n}^{\prime}(0)-\omega^{-} \beta_{n}(0) \geq \beta_{n}(0)\left[m \frac{e^{\omega^{-} t}-1}{\omega^{-}}-\omega^{-}\right] .
$$

Chosing $\tau_{0}$ large enough we get a positive right hand side for $t \geq \tau_{0}$, hence the left inequality in (3.17). The right inequality follows similarly by differentiating $\beta_{n}^{\prime}-\omega^{+} \beta_{n}$ and we omit the details of the proof.

Proof of (3.18). Assume as in the proof of (3.17) that $\beta_{n}(0) \geq 0$ and $\beta_{n}^{\prime}(0) \geq 0$. By (3.25), and using that $\beta_{n}$ is positive on $(0, T)$,

$$
\forall t \in\left[0, t_{n}\right], \quad \beta_{n}^{\prime \prime}(t)-\omega^{+2} \beta_{n}(t) \leq\left(\omega^{2}+m-\left(\omega^{+}\right)^{2}\right) \beta_{n}(t)<0 .
$$

This shows by a standard ODE argument that $\beta_{n}(t) \leq \tilde{\beta}_{n}(t)$, where $\tilde{\beta}_{n}(t)$ is the solution of the differential equation $\tilde{\beta}_{n}^{\prime \prime}-\omega^{+2} \tilde{\beta}_{n}=0$ with initial conditions $\tilde{\beta}_{n}(0)=\beta_{n}(0), \tilde{\beta}_{n}^{\prime}(0)=\beta_{n}^{\prime}(0)$. Hence

$$
\forall t \in\left[0, t_{n}\right], \quad \beta_{n}(t) \leq \beta_{n}(0) \cosh \left(\omega^{+} t\right)+\frac{\beta_{n}^{\prime}(0)}{\omega^{+}} \sinh \left(\omega^{+} t\right)
$$

By (3.7),

$$
\partial_{t} h_{n}(0)=\beta_{n}^{\prime}(0) \mathcal{Y}+\sum_{j=0}^{N} \gamma_{j, n}^{\prime}(0) W_{j}+g_{n}(0), \quad g_{n}(0) \in G_{\perp} .
$$

Taking the $L^{2}$-scalar product with $\mathcal{Y}$ and recalling that $W_{j}, j=0 \ldots N$, and $g_{n}(0)$ are orthogonal to $\mathcal{Y}$, we get $\left|\beta_{n}^{\prime}(0)\right| \leq\left\|\partial_{t} h_{n}(0)\right\|_{2}$. Thus, in view of Claim 3.6, for large $n$ :

$$
\beta_{n}^{\prime}(0) \leq\left(M_{0}+1\right) \beta_{n}(0) \text {. }
$$

By (3.26)

$$
\beta_{n}\left(\tau_{0}\right) \leq \beta_{n}(0) \cosh \left(\omega^{+} \tau_{0}\right)+\frac{M_{0}+1}{\omega^{+}} \beta_{n}(0) \sinh \left(\omega^{+} \tau_{0}\right) \leq K_{1} \beta_{n}(0),
$$

for some constant $K_{1}$ depending only on the choice of $\omega^{+}$. By (3.17),

$$
\forall t \geq \tau_{0}, \quad e^{\omega^{-}\left(t-\tau_{0}\right)} \beta_{n}\left(\tau_{0}\right) \leq \beta_{n}(t) \leq e^{\omega^{+}\left(t-\tau_{0}\right)} \beta_{n}\left(\tau_{0}\right) .
$$

Using (3.27) for the upper bound and the fact that $\beta_{n}$ increases for the lower bound, we get

$$
\forall t \geq \tau_{0}, \quad e^{\omega^{-}\left(t-\tau_{0}\right)} \beta_{n}(0) \leq \beta_{n}(t) \leq K_{1} e^{\omega^{+}\left(t-\tau_{0}\right)} \beta_{n}(0),
$$

which yields (3.18).

Proof of (3.19).

We divide the proof into two steps.

Step 1. Estimates on the coefficients

We first show that there exist a constant $C_{1}>0$, independent of the parameters $M$ and $\eta$, such that for all $t \in\left[0, t_{n}\right]$

$$
\begin{aligned}
\frac{1}{C_{1}}\left|\beta_{n}\right|-C_{1}\left\|\partial_{t, x} h_{n}\right\|_{2}^{3 / 2} & \leq\left\|\nabla g_{n}\right\|_{2}+\left\|\partial_{t} h_{n}\right\|_{2}+\varepsilon_{n} \leq C_{1} \beta_{n}+C_{1}\left\|\partial_{t, x} h_{n}\right\|_{2}^{3 / 2} \\
\frac{1}{C_{1}}\left\|\partial_{t, x} h_{n}\right\|_{2} & \leq\left|\beta_{n}\right|+\sum_{j=0}^{N}\left|\gamma_{j, n}\right| \leq C_{1}\left\|\partial_{t, x} h_{n}\right\|_{2}
\end{aligned}
$$


We have

$$
E\left(W+h_{n}, \partial_{t} h_{n}\right)=E(W, 0)-\varepsilon_{n}^{2}
$$

Thus there exists a constant $C_{2}>0$ (independent of the parameters) such that

$$
\left.\left|Q\left(h_{n}\right)+\int\right| \partial_{t} h_{n}\right|^{2}+\varepsilon_{n}^{2} \mid \leq C_{2}\left\|\partial_{t, x} h_{n}(t)\right\|_{2}^{3} .
$$

Furthermore, by (3.7) (and the fact that the functions $W_{j}, j=0 \ldots N$ are in the kernel of $Q$ )

$$
Q\left(h_{n}\right)=-\beta_{n}{ }^{2}|Q(\mathcal{Y})|+Q\left(g_{n}\right) .
$$

Which yields

$$
\left.\left|-\beta_{n}{ }^{2}\right| Q(\mathcal{Y})\left|+Q\left(g_{n}\right)+\int\right| \partial_{t} h_{n}\right|^{2}+\varepsilon_{n}^{2} \mid \leq C_{2}\left\|\partial_{t, x} h_{n}(t)\right\|_{2}^{3} .
$$

As $g_{n} \in G_{\perp}$, we have $Q\left(g_{n}\right) \approx\left\|\nabla g_{n}\right\|_{2}^{2}$, which yields (3.28).

Let us show (3.29). Note that the upper bound follows immediately from the definitions of $\beta_{n}$ and $\gamma_{j, n}$ (see (3.8) and (3.9) ). It remains to show the lower bound. We have

$$
h_{n}(t)=\beta_{n}(t) \mathcal{Y}+\sum_{j=0}^{N} \gamma_{j, n}(t) W_{j}+g_{n}(t)
$$

and hence, by $(3.28)$

$$
\begin{gathered}
\left\|\nabla h_{n}\right\|_{2} \leq C\left[\left|\beta_{n}\right|+\sum_{j=0}^{N}\left|\gamma_{j, n}\right|+\left\|\nabla g_{n}\right\|_{2}\right] \leq C\left[\left|\beta_{n}\right|+\sum_{j=0}^{N}\left|\gamma_{j, n}\right|+\left\|\partial_{t, x} h_{n}\right\|_{2}^{\frac{3}{2}}\right] \\
\left\|\partial_{t, x} h_{n}\right\|_{2}=\left\|\nabla h_{n}\right\|_{2}+\left\|\partial_{t} h_{n}\right\|_{2} \leq C\left[\left|\beta_{n}\right|+\sum_{j=0}^{N}\left|\gamma_{j, n}\right|+\left\|\partial_{t, x} h_{n}\right\|_{2}^{\frac{3}{2}}\right] .
\end{gathered}
$$

As a consequence of $(3.20)$ and (3.21), we obtain

$$
\left\|\partial_{t, x} h_{n}\right\|_{2}=\leq C\left[\left|\beta_{n}\right|+\sum_{j=0}^{N}\left|\gamma_{j, n}\right|\right]+C\left\|\partial_{t, x} h_{n}\right\|_{2} M^{1 / 2} \eta^{1 / 2} .
$$

by $(3.15)$, we get the lower bound in 3.29 )

Step 2. Bound on $\gamma_{j, n}$.

We are now ready to show (3.19). According to $(3.29)$, it is sufficient to show that there exists a constant $C_{3}$ independent of $M$ and $\eta \leq \frac{1}{K_{0} M^{3}}$ such that

$$
\forall j \in\{0, \ldots, N\}, \forall t \in\left[0, t_{n}\right], \quad\left|\gamma_{j, n}(t)\right| \leq C_{3}\left|\beta_{n}(t)\right| .
$$

We have, for $j=0 \ldots N$.

$$
\gamma_{j, n}^{\prime}(t)=\int \nabla\left(\partial_{t} h_{n}(t)-\beta_{n}{ }^{\prime}(t) \mathcal{Y}\right) \nabla W_{j}
$$

Note that $\int \nabla W_{j} \nabla \mathcal{Y}=0$ if $j \geq 1$, but we won't need this fact in the sequel. The preceding inequality yields

$$
\left|\gamma_{j, n}^{\prime}(t)\right| \leq C\left(\left\|\partial_{t} h_{n}(t)\right\|_{2}+\left|\beta_{n}^{\prime}(t)\right|\right)
$$


By (3.28) and assumptions (3.20) and (3.21),

$$
\left\|\partial_{t} h_{n}\right\|_{2} \leq C_{1}\left(\left|\beta_{n}\right|+\left\|\partial_{t, x} h_{n}\right\|_{2}^{3 / 2}\right) \leq C_{1}\left|\beta_{n}\right|\left(1+\eta^{1 / 2} M^{3 / 2}\right) .
$$

Taking $\eta$ small enough so that $\eta^{1 / 2} M^{3 / 2} \leq 1$, we get

$$
\left\|\partial_{t} h_{n}\right\|_{2} \leq 2 C_{1}\left|\beta_{n}\right|
$$

By (3.32), taking a larger constant $C$,

$$
\left|\gamma_{j, n}^{\prime}\right| \leq C\left(\left|\beta_{n}\right|+\left|\beta_{n}^{\prime}\right|\right) .
$$

Integrating between 0 and $t \leq \tau_{0}$, and using that $\gamma_{j, n}(0)=0$, that $\left|\beta_{n}\right|$ increases and that the sign of $\beta_{n}^{\prime}(t)$ is independant of $t \in\left[0, t_{n}\right]$ (see (3.24)), we obtain

$$
\forall t \in\left[0, \tau_{0}\right], \quad\left|\gamma_{j, n}(t)\right| \leq C(t+1)\left|\beta_{n}(t)\right| .
$$

This yields (3.31) for $t \leq \tau_{0}$.

Now by (3.17) and (3.34), and using that the signs of $\beta_{n}$ and $\beta_{n}^{\prime}$ do not depend on time,

$$
\forall t \geq \tau_{0}, \quad\left|\gamma_{j, n}^{\prime}(t)\right| \leq C\left|\beta_{n}{ }^{\prime}(t)\right| .
$$

Integrating between $\tau_{0}$ and $t \in\left[\tau_{0}, t_{n}\right]$, we get

$$
\left|\gamma_{j, n}(t)\right| \leq C\left(\left|\beta_{n}(t)\right|+\left|\gamma_{j, n}\left(\tau_{0}\right)\right|\right) .
$$

Using (3.35) at $t=\tau_{0}$ and the fact that $\left|\beta_{n}\right|$ increases, we get (3.31) for $t \geq \tau_{0}$. The proof is complete.

\section{Proof of main Result}

This section is devoted to the proof of Theorem 1. The proof is divided into 3 steps. In Step 1 , we show the lower bound, in the next two steps the upper bound.

Step 1. Lower bound.

We must show

$$
\liminf _{\varepsilon \rightarrow 0^{+}} \frac{\mathcal{I}_{\varepsilon}}{|\log \varepsilon|} \geq \frac{2}{\omega} \int_{\mathbb{R}^{N}} W^{\frac{2(N+1)}{N-2}}
$$

For this we first note that

$$
\int \nabla W \cdot \nabla \mathcal{Y}=-\int \Delta W \mathcal{Y}=\int W^{\frac{N+2}{N-2}} \mathcal{Y}>0
$$

as $\mathcal{Y}$ and $W$ are positive. Consider the family of solutions $\left(u^{a}\right)_{a>0}$ of (1.1) with initial conditions

$$
u_{0}^{a}=W-a \mathcal{Y}, \quad u_{1}^{a}=0 .
$$

For small $a>0$,

$$
\int\left|\nabla u_{0}^{a}\right|^{2}=\int|\nabla W|^{2}-2 a \int \nabla W \cdot \nabla \mathcal{Y}+a^{2} \int|\nabla \mathcal{Y}|^{2}<\int|\nabla W|^{2} .
$$

We have

$$
E\left(u_{0}^{a}, u_{1}^{a}\right)=E(W, 0)+Q(-a \mathcal{Y})+O\left(a^{3}\right)=E(W, 0)-a^{2}|Q(\mathcal{Y})|+O\left(a^{3}\right) .
$$


We argue by contradiction. If (4.1) does not hold, there exists a sequence $\varepsilon_{n}$ which tends to 0 such that for some $\rho>\omega$

$$
\forall n, \quad \frac{2}{\rho} \int_{\mathbb{R}^{N}} W^{\frac{2(N+1)}{N-2}} \geq \frac{\mathcal{I}_{\varepsilon_{n}}}{\left|\log \varepsilon_{n}\right|} .
$$

By (4.2), and using that $E\left(u_{0}^{a}, u_{1}^{a}\right)$ is a continuous function of $a$, there exists a sequence $a_{n}$ such that

Furthermore,

$$
\varepsilon_{n}^{2}=E(W, 0)-E\left(u_{0}^{a_{n}}, u_{1}^{a_{n}}\right),
$$

$$
\varepsilon_{n} \sim a_{n} \sqrt{|Q(\mathcal{Y})|} \text { as } n \rightarrow+\infty
$$

Let $u_{n}=u^{a_{n}}$. Observe that

$$
\partial_{t} u_{n}(0)=0, \quad\left\|\nabla\left(u_{n}(0)-W\right)\right\|_{2}=a_{n}\|\nabla \mathcal{Y}\|_{2} \underset{n \rightarrow+\infty}{\longrightarrow} 0 .
$$

Furthermore, $\beta_{n}(0)=-a_{n}, \beta_{n}^{\prime}(0)=0$, which shows that the assumptions of Proposition 3.4 are satisfied. Consider a small $\eta>0$. By Proposition 3.4

$$
\lim _{n \rightarrow+\infty} \frac{T_{n}(\eta)}{\left|\log a_{n}\right|}=\lim _{n \rightarrow+\infty} \frac{T_{n}(\eta)}{|\log | \beta_{n}(0)||}=\frac{1}{\omega}
$$

By (4.4),

$$
\lim _{n \rightarrow+\infty} \frac{T_{n}(\eta)}{|\log | \varepsilon_{n}||}=\frac{1}{\omega} .
$$

Let us give a lower bound for $\left\|u_{n}\right\|_{S(0,+\infty)}$. From now on we will write $T_{n}$ instead of $T_{n}(\eta)$ for the sake of simplicity. As $u_{n}=W+h_{n}$, we have

$$
\left\|u_{n}\right\|_{S\left(0, T_{n}\right)} \geq\|W\|_{S\left(0, T_{n}\right)}-\left\|h_{n}\right\|_{S\left(0, T_{n}\right)} .
$$

Furthermore,

Write

$$
\|W\|_{S\left(0, T_{n}\right)}=T_{n}^{\frac{N-2}{2(N+1)}}\|W\|_{\frac{2(N+1)}{N-2}} .
$$

$$
\|h\|_{S\left(0, T_{n}\right)}^{\frac{2(N+1)}{N-2}}=\sum_{I \in E_{T_{n}}}\|h\|_{S(I)}^{\frac{2(N+1)}{N-2}},
$$

where $E_{T_{n}}$ is a set of at most $\frac{T_{n}}{\tilde{c}}+1$ subinterval of $\left(0, T_{n}\right)$, of length at most $\tilde{c}$ (given by Lemma 3.2) such that $\left(0, T_{n}\right)=\bigcup_{I \in E_{T_{n}}} \bar{I}$. By Lemma 3.2 and the fact that $\left\|\nabla h_{n}\right\|_{2}+\left\|\partial_{t} h_{n}\right\|_{2} \leq M \eta$ on $\left(0, T_{n}\right)$, we get for small $\eta>0$,

$$
\left\|h_{n}\right\|_{S\left(0, T_{n}\right)}^{\frac{2(N+1)}{N-2}} \leq C\left(\frac{T_{n}}{\tilde{c}}+1\right) \eta^{\frac{2(N+1)}{N-2}} .
$$

Hence a constant $C>0$ such that

$$
\left\|h_{n}\right\|_{S\left(0, T_{n}\right)} \leq C \eta T_{n}^{\frac{N-2}{2(N+1)}} .
$$

Combining the preceding estimates, we obtain

$$
\int_{0}^{T_{n}} \int_{\mathbb{R}^{N}}\left|u_{n}\right|^{\frac{2(N+1)}{N-2}} \geq T_{n}\left[\|W\|_{\frac{2(N+1)}{N-2}}-C \eta\right]^{\frac{2(N+1)}{N-2}} .
$$


Hence with (4.5),

$$
\liminf _{n \rightarrow+\infty} \frac{1}{\left|\log \varepsilon_{n}\right|} \int_{0}^{+\infty} \int_{\mathbb{R}^{N}}\left|u_{n}\right|^{\frac{2(N+1)}{N-2}} \geq \frac{1}{\omega}\left[\|W\|_{\frac{2(N+1)}{N-2}}-C \eta\right]^{\frac{2(N+1)}{N-2}} .
$$

Letting $\eta$ tends to 0 we obtain

$$
\liminf _{n \rightarrow+\infty} \frac{1}{\left|\log \varepsilon_{n}\right|} \int_{0}^{+\infty}\left|u_{n}\right|^{\frac{2(N+1)}{N-2}} \geq \frac{1}{\omega}\|W\|_{\frac{2(N+1)}{N-2}}^{\frac{2(N+1)}{N-2}} .
$$

Next, notice that as $\partial_{t} u(0)=0$, the uniqueness in the Cauchy problem (1.1) implies $u(t, x)=$ $u(-t, x)$ and thus

Finally,

$$
\liminf _{n \rightarrow+\infty} \frac{1}{\left|\log \varepsilon_{n}\right|} \int_{-\infty}^{0}\left|u_{n}\right|^{\frac{2(N+1)}{N-2}} \geq \frac{1}{\omega}\|W\|_{\frac{2(N+1)}{N-2}}^{\frac{2(N+1)}{N-2}} .
$$

$$
\liminf _{n \rightarrow+\infty} \frac{\mathcal{I}_{\varepsilon_{n}}}{\left|\log \varepsilon_{n}\right|} \geq \liminf _{n \rightarrow+\infty} \frac{1}{\left|\log \varepsilon_{n}\right|} \int_{-\infty}^{+\infty}\left|u_{n}\right|^{\frac{2(N+1)}{N-2}} \geq \frac{2}{\omega}\|W\|_{\frac{2(N+1)}{N-2}}^{\frac{2(N+1)}{N-2}},
$$

contradicting (4.3). Step 1 is complete.

Step 2. Estimate before the exit time.

We next show the upper bound on $\mathcal{I}_{\varepsilon}$, i.e that

$$
\limsup _{\varepsilon \rightarrow 0^{+}} \frac{\mathcal{I}_{\varepsilon}}{|\log \varepsilon|} \leq \frac{2}{\omega} \int_{\mathbb{R}^{N}} W^{\frac{2(N+1)}{N-2}} .
$$

For this we will show that if $\varepsilon_{n}>0$ is a sequence that goes to 0 and $u_{n}$ a sequence of solutions of (1.1) such that

$$
\left\|\nabla u_{n}(0)\right\|_{2}<\|\nabla W\|_{2}, \quad E(W, 0)-E\left(u_{n}, \partial_{t} u_{n}\right)=\varepsilon_{n}^{2}
$$

then

$$
\limsup _{n \rightarrow+\infty} \frac{1}{\left|\log \varepsilon_{n}\right|} \int_{\mathbb{R}_{\mathbb{R}^{N}}}\left|u_{n}\right|^{\frac{2(N+1)}{N-2}} \leq \frac{2}{\omega} \int_{\mathbb{R}^{N}} W^{\frac{2(N+1)}{N-2}} .
$$

Possibly time-translating $u_{n}$, we may assume

$$
\left\|u_{n}\right\|_{S(-\infty, 0)}=\left\|u_{n}\right\|_{S(0,+\infty)} \underset{n \rightarrow+\infty}{\longrightarrow}+\infty
$$

By Proposition 2.2, rescaling and space-translating $u_{n}$ if necessary, we can assume

$$
\lim _{n \rightarrow+\infty} u_{n}=W \text {. }
$$

Consider the functions $h_{n}$ and $g_{n}$, and the parameters $\beta_{n}$ and $\gamma_{j, n}$ defined in the beginning of \$3.2. Replacing $u_{n}(x, t)$ by $u_{n}(x,-t)$ if it is not the case, we may assume

$$
\beta_{n}(0) \beta_{n}{ }^{\prime}(0) \geq 0 \text {. }
$$

Furthermore, by Claim 3.3, we may also assume (3.12).

Fix a small $\eta>0$, and consider $T_{n}=T_{n}(\eta)$ defined by Proposition 3.4. In this step, we show that there exists a constant $C>0$ such that

$$
\limsup _{n \rightarrow+\infty} \frac{1}{\left|\log \varepsilon_{n}\right|} \int_{0}^{T_{n}(\eta)} \int_{\mathbb{R}^{N}}\left|u_{n}\right|^{\frac{2(N+1)}{N-2}} \leq \frac{1}{\omega}\left[\|W\|_{\frac{2(N+1)}{N-2}}+C \eta\right]^{\frac{2(N+1)}{N-2}} .
$$

Indeed, by Claim 3.6, for large $n$,

$$
\varepsilon_{n} \leq M_{0}\left|\beta_{n}(0)\right|
$$


Hence by Proposition 3.4,

$$
\limsup _{n \rightarrow+\infty} \frac{T_{n}}{\left|\log \varepsilon_{n}\right|} \leq \frac{1}{\omega} .
$$

By the same argument as in Step 1, we get

$$
\int_{0}^{T_{n}} \int_{\mathbb{R}^{N}}\left|u_{n}\right|^{\frac{2(N+1)}{N-2}} \leq T_{n}\left[\|W\|_{\frac{2(N+1)}{N-2}}+C \eta\right]^{\frac{2(N+1)}{N-2}} .
$$

Hence

$$
\limsup _{n \rightarrow+\infty} \frac{1}{T_{n}} \int_{0}^{T_{n}} \int_{\mathbb{R}^{N}}\left|u_{n}\right|^{\frac{2(N+1)}{N-2}} \leq\left[\|W\|_{\frac{2(N+1)}{N-2}}+C \eta\right]^{\frac{2(N+1)}{N-2}} .
$$

Combining with (4.12), we obtain (4.11).

Step 3. Estimate for large time.

To conclude the proof, we will show that if $\eta$ is small enough, there exists a constant $C(\eta)>0$ such that for large $n$

$$
\left\|u_{n}\right\|_{S\left(T_{n}(\eta),+\infty\right)} \leq C(\eta) .
$$

Assuming (4.13), we obtain by (4.11),

$$
\limsup _{n \rightarrow+\infty} \frac{1}{\left|\log \varepsilon_{n}\right|}\left\|u_{n}\right\|_{S(0,+\infty)}^{\frac{2(N+1)}{N-2}}=\limsup _{n \rightarrow+\infty} \frac{1}{\left|\log \varepsilon_{n}\right|}\left\|u_{n}\right\|_{S\left(0, T_{n}(\eta)\right)}^{\frac{2(N+1)}{N-2}} \leq \frac{1}{\omega}\left[\|W\|_{\frac{2(N+1)}{N-2}}+C \eta\right]^{\frac{2(N+1)}{N-2}} .
$$

Letting $\eta$ tend to 0 we get

$$
\limsup _{n \rightarrow+\infty} \frac{1}{\left|\log \varepsilon_{n}\right|}\left\|u_{n}\right\|_{S(0,+\infty)}^{\frac{2(N+1)}{N-2}} \leq \frac{1}{\omega}\|W\|_{\frac{2(N+1)}{N-2}}^{\frac{2(N+1)}{N-2}},
$$

which shows, in view of (4.9), the desired estimate (4.8).

It remains to show (4.13). We will argue by contradiction. If (4.13) does not hold, there exist a subsequence of $\left(u_{n}\right)$, still denoted by $\left(u_{n}\right)$ such that

$$
\left\|u_{n}\right\|_{S\left(T_{n},+\infty\right) \underset{n \rightarrow \infty}{\longrightarrow}+\infty}^{\longrightarrow}
$$

Furthermore, by (4.9)

$$
\left\|u_{n}\right\|_{S\left(-\infty, T_{n}\right)} \geq\left\|u_{n}\right\|_{S(-\infty, 0)}=\left\|u_{n}\right\|_{S(0,+\infty)} \underset{n \rightarrow \infty}{\longrightarrow}+\infty .
$$

In view of (4.14) and (4.15), Proposition 2.2 (国) implies that there exists sequences $\lambda_{n}>0$, $x_{n} \in \mathbb{R}^{N}$, and $\delta_{0} \in\{-1,+1\}$ such that

$$
\lim _{n \rightarrow+\infty}\left\|\frac{\delta_{0}}{\lambda_{n}^{N / 2}} \nabla u_{n}\left(T_{n}, \frac{-x_{n}}{\lambda_{n}}\right)-\nabla W\right\|_{2}+\left\|\frac{\partial u_{n}}{\partial t}\left(T_{n}\right)\right\|_{2}=0 .
$$

By Proposition 3.4,

$$
\liminf _{n \rightarrow+\infty}\left|\beta_{n}^{\prime}\left(T_{n}\right)\right| \geq \omega \eta
$$

By the decomposition (3.7) of $h_{n}$,

$$
\int \partial_{t} u_{n}\left(T_{n}\right) \mathcal{Y}=\int \partial_{t} h_{n}\left(T_{n}\right) \mathcal{Y}=\beta_{n}^{\prime}\left(T_{n}\right)
$$


This shows by (4.16) that $\beta_{n}^{\prime}\left(T_{n}\right)$ must tend to 0 , yielding a contradiction. This concludes the proof of (4.13) and thus of Theorem 1 .

\section{ESTIMATE OF THE SCATTERING NORM FOR ENERGY-CRITICAL FOCUSING NLS}

In this section we briefly adress the case of the radial energy critical focusing semilinear Schrödinger equation

$$
i \partial_{t} u+\Delta u+|u|^{\frac{4}{N-2}} u=0, \quad u_{\mid t=0}=u_{0} \in \dot{H}_{r}^{1},
$$

where $N \in\{3,4,5\}$ and $\dot{H}_{r}^{1}$ is the subset of $\mathbb{R}^{N}$ of spherically symmetric functions. The equation (5.1) is locally well-posed (see CW90]) in the energy space $\dot{H}_{r}^{1}$. Furthermore, if $I_{\max } \ni 0$ is the maximal interval of definition then

$$
J \Subset I_{\max } \Longrightarrow\|u\|_{\tilde{S}(J)}<\infty, \text { where } \tilde{S}(J)=L^{\frac{2(N+2)}{N-2}},
$$

and globally defined solutions of (5.1) such that $\|u\|_{\tilde{S}(\mathbb{R})}$ is finite scatter (see Bou99b, Bou99a]).

The energy

is conserved.

$$
\mathcal{E}(u(t))=\frac{1}{2} \int|\nabla u(t)|^{2}-\frac{N-2}{2 N} \int|u(t)|^{\frac{2 N}{N-2}}
$$

In the defocusing case, all solutions are known to be globally defined and scatter Bou99b, Tao05. Furthermore, in Tao05, T. Tao gave a bound of $\|u\|_{\tilde{S}(\mathbb{R})}$ in term of an exponential of a power of the conserved defocusing energy $\frac{1}{2} \int\left|\nabla u_{0}\right|^{2}+\frac{N-2}{2 N} \int|u|^{\frac{2 N}{N-2}}$.

In the focusing case, the function $W$, defined in (1.2) is still a stationnary solution of $W$. The following theorem shown in KM06a for the case $\mathcal{E}\left(u_{0}\right)<\mathcal{E}(W)$ and in DM07a for the case $\mathcal{E}\left(u_{0}\right)=\mathcal{E}(W)$, is the analoguous of Theorem $\mathrm{A}$ for equation (5.1).

Theorem B (Kenig-Merle,Duyckaerts-Merle). There exists a global solution $\widetilde{W}_{-}$of (5.1) such that

$$
\begin{gathered}
\mathcal{E}\left(\widetilde{W}^{-}\right)=\mathcal{E}(W), \quad\left\|\nabla \widetilde{W}_{-}(0)\right\|_{2}<\|\nabla W\|_{2} \\
\left\|\widetilde{W}^{-}\right\|_{\tilde{S}(-\infty, 0)}<\infty, \quad \lim _{t \rightarrow+\infty}\left\|\nabla\left(\widetilde{W}^{-}(t)-W\right)\right\|_{2}=0 .
\end{gathered}
$$

Moreover, if $u$ is a radial solution of (5.1) such that $\mathcal{E}\left(u_{0}\right) \leq \mathcal{E}(W)$ and $\left\|\nabla u_{0}\right\|_{2} \leq\|\nabla W\|_{2}$, then $u$ is globally defined. If furthermore $\|u\|_{\tilde{S}(\mathbb{R})}=\infty$, then $u=\widetilde{W}^{-}$or $u=W$ up to the invariances of the equation.

Defining

$$
\tilde{F}_{\varepsilon}:=\left\{u \text { radial solution of (5.1) such that } \mathcal{E}\left(u_{0}\right) \leq \mathcal{E}(W)-\varepsilon^{2} \text { and } \int\left|\nabla u_{0}\right|^{2}<\int|\nabla W|^{2}\right\} \text {. }
$$

we get in particular that for $\varepsilon>0$ the supremum

$$
\widetilde{\mathcal{I}}_{\varepsilon}=\sup _{u \in \tilde{F}_{\varepsilon}} \int_{\mathbb{R} \times \mathbb{R}^{N}}|u(t, x)|^{\frac{2(N+2)}{N-2}} d t d x=\sup _{u \in \tilde{F}_{\varepsilon}}\|u\|_{\tilde{S}(\mathbb{R})}^{\frac{2(N+2)}{N-2}}
$$

is finite, and that

$$
\lim _{\varepsilon \rightarrow 0^{+}} \widetilde{\mathcal{I}}_{\varepsilon}=+\infty
$$


We wish again to estimate of $\widetilde{\mathcal{I}}_{\varepsilon}$ when $\varepsilon$ goes to 0 . As in the case of the wave equation, the behavior of $\widetilde{\mathcal{I}}_{\varepsilon}$ is determined by the linearized operator near $W$. If $u=W+h$ is a solution of (5.1), then, identifying $h$ with the column vector $(\operatorname{Re} h, \operatorname{Im} h)^{T}=\left(h_{1}, h_{2}\right)^{T}$.

$$
\partial_{t} h+\mathcal{L}(h)+R(h)=0, \quad \mathcal{L}:=\left(\begin{array}{cc}
0 & \Delta+W^{\frac{4}{N-2}} \\
-\Delta-\frac{N+2}{N-2} W^{\frac{4}{N-2}} & 0
\end{array}\right),
$$

where an appropriate norm of $R(h)$ is bounded by $\|\nabla h\|_{2}^{2}$ when $h$ is small. It is known (see DM07a, Section 7.1]) that the essential spectrum of $\mathcal{L}$ is $i \mathbb{R}$ and that $\mathcal{L}$ admits only two nonzero real eigenvalues, $\widetilde{\omega}>0$ and $-\widetilde{\omega}$, with eigenfunctions $\widetilde{\mathcal{Y}}_{ \pm}$which are in the space of Schwartz functions. Then:

\section{Theorem 2.}

$$
\lim _{\varepsilon \rightarrow 0^{+}} \frac{\widetilde{\mathcal{I}}_{\varepsilon}}{|\log \varepsilon|}=\frac{2}{\widetilde{\omega}} \int_{\mathbb{R}^{N}} W^{\frac{2(N+2)}{N-2}}
$$

Our result is restricted to the radial case in spatial dimensions $N \in\{3,4,5\}$. In view of the recent work [KV08 on non-radial energy-critical focusing NLS in dimension $N \geq 5$, it is natural to expect that the same estimate holds in a more general situation.

The proof of Theorem 2 is very similar to the one of Theorem 1, and we will only sketch it, highlighting the minor differences. In $\$ 5.1$ we recall a few facts about the operator $\mathcal{L}$ and state without proof the analoguous of Propositions 2.2, Propositions 3.4 and Claim 3.6. In $\$ 5.2$ we briefly explain how to use these results to show Theorem 2 .

5.1. Convergence to $W$ and estimate on the exit time. In view of Theorem $\mathbb{B}$, and the use of the profile decomposition method in [KM06a, Section 4] (see also [DM07a, Lemma 2.5]), the proof of Proposition 5.1 adapts easily to show:

Proposition 5.1. Let $u_{n}$ be a family of radial solutions of (5.1), such that

$$
\mathcal{E}\left(u_{n}(0)\right)<\mathcal{E}(W), \quad\left\|\nabla u_{n}(0)\right\|_{2}<\|\nabla W\|_{2} .
$$

and $\lim _{n \rightarrow+\infty}\left\|u_{n}\right\|_{\tilde{S}(\mathbb{R})}=+\infty$. Let $\left(t_{n}\right)_{n}$ be a time sequence. Assume

$$
\lim _{n \rightarrow+\infty}\left\|u_{n}\right\|_{\tilde{S}\left(-\infty, t_{n}\right)}=\lim _{n \rightarrow+\infty}\left\|u_{n}\right\|_{\tilde{S}\left(t_{n},+\infty\right)}=+\infty .
$$

Then, up to the extraction of a subsequence, there exist $\theta_{0} \in \mathbb{R}$ and a sequence of parameters $\lambda_{n}>0$ such that

$$
\lim _{n \rightarrow+\infty}\left\|\frac{e^{i \theta_{0}}}{\lambda_{n}^{N / 2}} \nabla u_{n}\left(t_{n}, \frac{\cdot}{\lambda_{n}}\right)-\nabla W\right\|_{2}=0 .
$$

We next recall some spectral properties of the operator $\mathcal{L}$. We refer to [DM07a, $\S 5.1]$ for the details. We will often identify a complex-valued function $f$ with an $\mathbb{R}^{2}$-valued function $\left(f_{1}, f_{2}\right)^{T}$, with $f_{1}=\operatorname{Re} f, f_{2}=\operatorname{Im} f$. Developping the energy around $W$, we get, for small functions $h \in \dot{H}^{1}$,

$$
\mathcal{E}(W+h)=\mathcal{E}(W)+\widetilde{Q}(h)+O\left(\|h\|_{\frac{2 N}{N-2}}^{3}\right),
$$

where $\widetilde{Q}$ is the quadratic form $\widetilde{Q}(h)=B(h, h)$ and $B$ is defined by

$$
B(g, h)=\frac{1}{2} \int \nabla g_{1} \cdot \nabla h_{1}-\frac{N+2}{2(N-2)} \int g_{1} h_{1} W^{\frac{4}{N-2}}+\frac{1}{2} \int \nabla g_{2} \cdot \nabla h_{2}-\frac{1}{2} \int g_{2} h_{2} W^{\frac{4}{N-2}} .
$$


Denote by $\mathcal{Y}_{+}$the eigenfunction of $\mathcal{L}$ for the eigenvalue $\widetilde{\omega}$ and $\mathcal{Y}_{-}=m \overline{\mathcal{Y}}_{+}$the eigenfunction of $\mathcal{L}$ for the eigenvalue $-\widetilde{\omega}\left(m \neq 0\right.$ is a real normalization constant), and recall the definition of $W_{0}$ in (3.2). One may show that $W_{0}$ and $i W$ are in the kernel of $\widetilde{Q}$. Furthermore, $\widetilde{Q}\left(\mathcal{Y}_{+}\right)=\widetilde{Q}\left(\mathcal{Y}_{-}\right)=0$ and we may chose $m$ such that $B\left(\mathcal{Y}_{+}, \mathcal{Y}_{-}\right)=-1$. Let

$$
\tilde{G}_{\perp}:=\left\{h \in \dot{H}^{1}: \int \nabla W \cdot \nabla h_{2}=\int \nabla W_{0} \cdot \nabla h_{1}=B\left(\mathcal{Y}_{+}, h\right)=B\left(\mathcal{Y}_{-}, h\right)=0\right\} .
$$

By DM07a, Lemma 5.2], there exists a constant $c>0$ such that

$$
\forall h \in \tilde{G}_{\perp}, \quad Q(h) \geq c\|\nabla h\|_{2}^{2} .
$$

We consider as in $\$ 3.2$ a sequence $u_{n}$ of radial solutions of (5.1) such that

$$
\begin{gathered}
\mathcal{E}\left(u_{n}\right) \leq \mathcal{E}(W)-\varepsilon_{n}^{2}, \quad\left\|\nabla u_{n}(0)\right\|_{2}<\|\nabla W\|_{2}, \\
\lim _{n \rightarrow+\infty}\left\|\nabla u_{n}(0)-\nabla W\right\|_{2}=0,
\end{gathered}
$$

and develop $h_{n}=u_{n}-W$ as follows

$$
h_{n}(t)=\beta_{n}^{+}(t) \mathcal{Y}_{+}+\beta_{n}^{-}(t) \mathcal{Y}_{-}+\gamma_{n}(t) W_{0}+\delta_{n}(t) i W+g_{n}(t), \quad g_{n}(t) \in \tilde{G}_{\perp} .
$$

Arguing as in Claim 3.3, we may assume

$$
\gamma_{n}(0)=\delta_{n}(0)=0
$$

Then we have the following analog of Propositions 3.4 and Claim 3.6. We skip the proofs, that are very similar to the previous ones.

Proposition 5.2. There exist a constant $\eta_{0}$, such that for all $\eta \in\left(0, \eta_{0}\right)$, for all sequence $\left(u_{n}\right)$ satisfying (5.3), (5.4) and (5.6) if

$$
\begin{aligned}
& T_{n}^{+}(\eta)=\inf \left\{t \geq 0:\left|\beta_{n}^{-}(t)\right| \geq \eta\right\} \\
& T_{n}^{-}(\eta)=-\sup \left\{t \leq 0:\left|\beta_{n}^{+}(t)\right| \geq \eta\right\} .
\end{aligned}
$$

then for large $n, T_{n}^{+}(\eta)$ and $T_{n}^{-}(\eta)$ are finite and

$$
\lim _{n \rightarrow+\infty} \frac{T_{n}^{+}(\eta)}{\log \left|\beta_{n}^{-}(0)\right|}=\lim _{n \rightarrow+\infty} \frac{T_{n}^{-}(\eta)}{\log \left|\beta_{n}^{+}(0)\right|}=\frac{1}{\widetilde{\omega}} .
$$

Furthermore,

$$
\liminf _{n \rightarrow+\infty}\left|\beta_{n}^{\prime}\left(T_{n}^{+}(\eta)\right)\right| \geq \frac{\eta \widetilde{\omega}}{2}, \quad \liminf _{n \rightarrow+\infty}\left|\beta_{n}^{\prime}\left(T_{n}^{-}(\eta)\right)\right| \geq \frac{\eta \widetilde{\omega}}{2} .
$$

Observe that in contrast with the wave equation case, there are two eigenfunctions, and that we have distinguished between the coefficient $\beta_{n}^{-}$of $\mathcal{Y}_{-}$, which tends to grow for positive times, and the one of $\mathcal{Y}_{+}$, which plays a similar role for negative times.

Claim 5.3. There exists $M_{0}>0$ such that for all sequence $\left(u_{n}\right)$ of solutions of (5.1) satisfying (5.3), (5.4) and (5.6) we have

$$
\beta_{n}^{+} \beta_{n}^{-}(0) \neq 0 \text { and } \limsup _{n \rightarrow+\infty} \frac{\varepsilon_{n}^{2}}{\left|\beta_{n}^{+}(0) \beta_{n}^{-}(0)\right|} \leq M_{0} .
$$




\subsection{Sketch of the proof of Theorem 2.}

Step 1. Lower bound.

We first show

$$
\liminf _{\varepsilon \rightarrow 0^{+}} \frac{\widetilde{\mathcal{I}}_{\varepsilon}}{|\log \varepsilon|} \geq \frac{2}{\widetilde{\omega}} \int_{\mathbb{R}^{N}} W^{\frac{2(N+2)}{N-2}} .
$$

Multiplying $\mathcal{Y}_{+}$and $\mathcal{Y}_{-}$by -1 if necessary, we may assume $\operatorname{Re} \int \nabla W \cdot \nabla \mathcal{Y}_{ \pm}>0$. Consider the family of solutions $\left(u^{a}\right)_{a>0}$ of (5.1) with initial conditions

$$
u_{0}^{a}=W-a \mathcal{Y}_{+}-a \mathcal{Y}_{-} .
$$

Then for small $a>0, \int\left|\nabla u_{0}^{a}\right|^{2}<\int|\nabla W|^{2}$. Furthermore

$$
\mathcal{E}\left(u_{0}^{a}\right)=\mathcal{E}(W)-2 a^{2}+O\left(a^{3}\right) .
$$

We argue by contradiction. If (5.9) does not hold, there exists a sequence $\varepsilon_{n}$ which tends to 0 such that for some $\rho>\widetilde{\omega}$

$$
\forall n, \quad \frac{2}{\rho} \int_{\mathbb{R}^{N}} W^{\frac{2(N+2)}{N-2}} \geq \frac{\widetilde{\mathcal{I}}_{\varepsilon_{n}}}{\left|\log \varepsilon_{n}\right|} .
$$

We then chose a sequence $a_{n}$ such that

$$
\varepsilon_{n}^{2}=\mathcal{E}(W)-\mathcal{E}\left(u_{0}^{a_{n}}\right), \quad \varepsilon_{n}^{2} \sim 2 a_{n}^{2} \text { as } n \rightarrow+\infty .
$$

Let $u_{n}=u^{a_{n}}$. Then the assumptions of Proposition 5.2 are satisfied. Consider a small $\eta>0$. By Proposition 5.2, noting that $\beta_{n}^{+}(0)=\beta_{n}^{-}(0)=-a_{n}$, we get

$$
\lim _{n \rightarrow+\infty} \frac{T_{n}^{+}(\eta)}{\left|\log a_{n}\right|}=\lim _{n \rightarrow+\infty} \frac{T_{n}^{-}(\eta)}{\left|\log a_{n}\right|}=\frac{1}{\widetilde{\omega}}
$$

By (5.12),

$$
\lim _{n \rightarrow+\infty} \frac{T_{n}^{-}(\eta)}{|\log | \varepsilon_{n}||}=\lim _{n \rightarrow+\infty} \frac{T_{n}^{+}(\eta)}{|\log | \varepsilon_{n}||}=\frac{1}{\widetilde{\omega}} .
$$

Writing $u_{n}=W+h_{n}$, and arguing as in Step 1 of Section $\mathbb{Q}$, we obtain

$$
\int_{0}^{T_{n}^{+}(\eta)} \int_{\mathbb{R}^{N}}\left|u_{n}\right|^{\frac{2(N+2)}{N-2}} \geq T_{n}^{+}(\eta)\left[\|W\|_{\frac{2(N+2)}{N-2}}-C \eta\right]^{\frac{2(N+2)}{N-2}} .
$$

Hence with (5.13), and letting $\eta$ tends to 0 ,

$$
\liminf _{n \rightarrow+\infty} \frac{1}{\left|\log \varepsilon_{n}\right|} \int_{0}^{+\infty}\left|u_{n}\right|^{\frac{2(N+2)}{N-2}} \geq \frac{1}{\widetilde{\omega}}\|W\|_{\frac{2(N+2)}{N-2}}^{\frac{2(N+2)}{N-2}} .
$$

Arguing similarly for negative time, we obtain

$$
\liminf _{n \rightarrow+\infty} \frac{\widetilde{\mathcal{I}}_{\varepsilon_{n}}}{\left|\log \varepsilon_{n}\right|} \geq \liminf _{n \rightarrow+\infty} \frac{1}{\left|\log \varepsilon_{n}\right|} \int_{-\infty}^{+\infty}\left|u_{n}\right|^{\frac{2(N+2)}{N-2}} \geq \frac{2}{\widetilde{\omega}}\|W\|_{\frac{2(N+2)}{N-2}}^{\frac{2(N+2)}{N-2}},
$$

contradicting (5.11). Step 1 is complete.

Step 2. Upper bound.

To show the upper bound on $\widetilde{\mathcal{I}}_{\varepsilon}$, we must show that for any sequence $\varepsilon_{n}>0$ that goes to 0 any sequence $u_{n}$ of solutions of (5.1) such that

$$
\left\|\nabla u_{n}(0)\right\|_{2}<\|\nabla W\|_{2}, \quad \mathcal{E}(W)-\mathcal{E}\left(u_{n}\right)=\varepsilon_{n}^{2},
$$


we have

$$
\limsup _{n \rightarrow+\infty} \frac{1}{\left|\log \varepsilon_{n}\right|} \int_{\mathbb{R} \times \mathbb{R}^{N}}\left|u_{n}\right|^{\frac{2(N+2)}{N-2}} \leq \frac{2}{\widetilde{\omega}} \int_{\mathbb{R}^{N}} W^{\frac{2(N+2)}{N-2}} .
$$

In view of Proposition 5.1 and the analoguous of Claim 3.3, we may assume that $u_{n}$ satisfy the assumptions of Proposition 5.2.

Fix a small $\eta>0$, and consider $T_{n}^{ \pm}(\eta)$ defined by Proposition 5.2. Then by the same proof than in Step 2 of Section 1 , one can show that there exists a constant $C>0$ such that

$$
\begin{aligned}
& \limsup _{n \rightarrow+\infty} \frac{1}{|\log | \beta_{n}^{-}(0)||} \int_{0}^{T_{n}^{+}(\eta)} \int_{\mathbb{R}^{N}}\left|u_{n}\right|^{\frac{2(N+2)}{N-2}} \leq \frac{1}{\widetilde{\omega}}\left[\|W\|_{\frac{2(N+2)}{N-2}}+C \eta\right]^{\frac{2(N+2)}{N-2}} \\
& \limsup _{n \rightarrow+\infty} \frac{1}{|\log | \beta_{n}^{+}(0)||} \int_{T_{n}^{-}(\eta)}^{0} \int_{\mathbb{R}^{N}}\left|u_{n}\right|^{\frac{2(N+2)}{N-2}} \leq \frac{1}{\widetilde{\omega}}\left[\|W\|_{\frac{2(N+2)}{N-2}}+C \eta\right]^{\frac{2(N+2)}{N-2}} .
\end{aligned}
$$

By Claim 5.3, for large $n$,

$$
2\left|\log \varepsilon_{n}\right| \geq|\log | \beta_{n}^{-}(0)|+\log | \beta_{n}^{+}(0)||+o_{n}(1),
$$

which yields

$$
\limsup _{n \rightarrow+\infty} \int_{-T_{n}^{-}(\eta)}^{T_{n}^{+}(\eta)} \int_{\mathbb{R}^{N}}\left|u_{n}\right|^{\frac{2(N+2)}{N-2}} \leq \frac{2\left|\log \varepsilon_{n}\right|}{\widetilde{\omega}}\left[\|W\|_{\frac{2(N+2)}{N-2}}+C \eta\right]^{\frac{2(N+2)}{N-2}}
$$

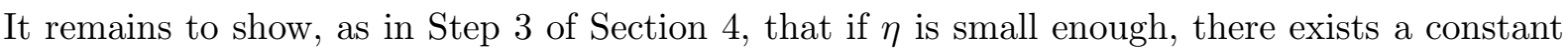
$C(\eta)>0$ such that for large $n$

$$
\left\|u_{n}\right\|_{S\left(-\infty,-T_{n}^{-}(\eta)\right)}+\left\|u_{n}\right\|_{S\left(T_{n}^{+}(\eta),+\infty\right)} \leq C(\eta) .
$$

Combining (5.16) and (5.17) and letting $\eta$ tends to 0 we would get (5.15).

To show (5.17), we argue by contradiction. Assume that there exists a subsequence of $\left(u_{n}\right)$, such that (from now on, we will write $T_{n}^{+}=T_{n}^{+}(\eta)$ )

$$
\left\|u_{n}\right\|_{S\left(T_{n}^{+},+\infty\right) \underset{n \rightarrow \infty}{\longrightarrow}+\infty}+
$$

Then by Proposition 5.1, there exists $\theta_{0} \in \mathbb{R}$ and a sequence $\lambda_{n}>0$, such that

$$
\lim _{n \rightarrow+\infty}\left\|\frac{e^{i \theta_{0}}}{\lambda_{n}^{N / 2}} \nabla u_{n}\left(T_{n}^{+}, \frac{\cdot}{\lambda_{n}}\right)-\nabla W\right\|_{2}=0 .
$$

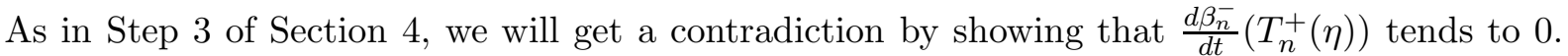
Unlike the case of the wave equation, the convergence to 0 of the time derivative of $u$ is not given directly by the compactness argument of Proposition 5.1. However, (5.18) and the fact that $u_{n}$ is a solution of $(5.1)$ which is in $C^{0}\left(\mathbb{R}, \dot{H}^{1}\right)$ shows that

$$
\lim _{n \rightarrow+\infty}\left\|\partial_{t} u_{n}\left(T_{n}^{+}\right)\right\|_{H^{-1}}=0 .
$$

As $\mathcal{Y}_{+}$is a Schwartz function (see DM07a, $\left.\S 7.2 .2\right]$ ), we get, at the point $t=T_{n}^{+}$,

$$
\left.\lim _{n \rightarrow+\infty} \frac{d}{d t} B\left(u_{n}(t), \mathcal{Y}_{+}\right)\right)=0 \text {. }
$$


The condition $g_{n}(t) \in G_{\perp}$ implies that $\beta_{n}^{-}(t)=-B\left(u_{n}(t)-W, \mathcal{Y}_{+}\right)$. Thus (5.8) contradicts (5.19). This shows

$$
\left\|u_{n}\right\|_{S\left(T_{n}^{+}(\eta),+\infty\right)} \leq C(\eta) .
$$

By a similar argument for negative time, we get (5.17). Combining (5.16) and (5.17), we obtain (5.15), which concludes the sketch of the proof of Theorem 2.

\section{REFERENCES}

[BG99] Hajer Bahouri and Patrick Gérard. High frequency approximation of solutions to critical nonlinear wave equations. Amer. J. Math., 121(1):131-175, 1999.

[Bou99a] J. Bourgain. Global solutions of nonlinear Schrödinger equations, volume 46 of American Mathematical Society Colloquium Publications. American Mathematical Society, Providence, RI, 1999.

[Bou99b] J. Bourgain. Global wellposedness of defocusing critical nonlinear Schrödinger equation in the radial case. J. Amer. Math. Soc., 12(1):145-171, 1999.

[CW90] Thierry Cazenave and Fred B. Weissler. The Cauchy problem for the critical nonlinear Schrödinger equation in $H^{s}$. Nonlinear Anal., 14(10):807-836, 1990.

[DM07a] T. Duyckaerts and F. Merle. Dynamic of threshold solutions for energy-critical nls. To be published in Geom. Funct. Anal., 2007.

[DM07b] T. Duyckaerts and F. Merle. Dynamic of threshold solutions for energy-critical wave equation. To be published in Int. Math. Res. Not. IMRN, 2007.

[GSV92] J. Ginibre, A. Soffer, and G. Velo. The global Cauchy problem for the critical nonlinear wave equation. J. Funct. Anal., 110(1):96-130, 1992.

[GV95] J. Ginibre and G. Velo. Generalized Strichartz inequalities for the wave equation. J. Funct. Anal., 133(1):50-68, 1995.

[KM06a] Carlos E. Kenig and Frank Merle. Global well-posedness, scattering and blow-up for the energy-critical, focusing, non-linear Schrödinger equation in the radial case. Invent. Math., 166(3):645-675, 2006.

[KM06b] Carlos E. Kenig and Frank Merle. Global well-posedness, scattering and blow-up for the energy-critical focusing non-linear wave equation. To be published in Acta Math., 2006.

[KV08] Rowan Killip and Monica Vişan. The focusing energy-critical nonlinear schrödinger equation in dimensions five and higher. Preprint, 2008.

[LS95] Hans Lindblad and Christopher D. Sogge. On existence and scattering with minimal regularity for semilinear wave equations. J. Funct. Anal., 130(2):357-426, 1995.

[Pec84] Hartmut Pecher. Nonlinear small data scattering for the wave and Klein-Gordon equation. Math. Z., 185(2):261-270, 1984.

[SS94] Jalal Shatah and Michael Struwe. Well-posedness in the energy space for semilinear wave equations with critical growth. Internat. Math. Res. Notices, (7):303ff., approx. 7 pp. (electronic), 1994.

[SS98] Jalal Shatah and Michael Struwe. Geometric wave equations, volume 2 of Courant Lecture Notes in Mathematics. New York University Courant Institute of Mathematical Sciences, New York, 1998.

[Tao05] Terence Tao. Global well-posedness and scattering for the higher-dimensional energy-critical nonlinear Schrödinger equation for radial data. New York J. Math., 11:57-80 (electronic), 2005.

[Tao06] Terence Tao. Spacetime bounds for the energy-critical nonlinear wave equation in three spatial dimensions. Dyn. Partial Differ. Equ., 3(2):93-110, 2006.

E-mail address: thomas.duyckaerts@u-cergy.fr

Thomas Duyckaerts, Université de Cergy-Pontoise, Département de Mathématiques, Site de Saint Martin, 2 avenue Adolphe-Chauvin, 95302 Cergy-Pontoise cedex, France. 